\title{
EL PENSAMIENTO DEL PROFESORADO EN CICLOS FORMATIVOS DE TURISMO RESPECTO A LA LABOR DOCENTE
}

\author{
Ana María de la Torre Frade ${ }^{1}$ \\ INS Escola d'Hoteleria i Turisme de Barcelona \\ atorre47@xtec.cat
}

\section{Resumen}

(Fecha envío: 24/04/17 - Fecha aceptación:)

\begin{abstract}
Esta investigación pretende constatar la realidad actual sobre las creencias y opiniones del profesorado de ciclos formativos de turismo que trabajan en centros públicos situados en la comunidad de Cataluña. Se plantean cuestiones como la satisfacción del profesorado, sus preocupaciones respecto a la educación y las problemáticas propias de la profesión docente. Intenta indagar cuál es su pensamiento sobre la labor docente, los desafíos a los que se enfrenta, la importancia de la formación, el grado de satisfacción y las condiciones laborales entre muchos otros temas.
\end{abstract}

\section{Palabras clave}

pensamiento del profesor, ciclos formativos, turismo, creencias del profesorado, mejora de la enseñanza

\begin{abstract}
This research aims to verify the current reality about the beliefs and opinions of the teaching staff of Tourism Training Cycles that work in public centers located in the community of Catalonia. Issues such as teacher satisfaction, concerns about education and problems of the teaching profession are raised. He tries to investigate his thoughts about the quality of teaching, the challenges of the teacher, the importance of training, the degree of satisfaction and working conditions among many other topics.
\end{abstract}

\section{Key words}

teaching thoughts, vocational education and training, tourism, teacher Beliefs, improvement of teaching

\section{INTRODUCCIÓN}

La crisis que vive nuestra sociedad no solo se refiere al ámbito económico, laboral, medioambiental y sociocultural, sino que afecta también a la enseñanza y la pérdida de valores humanos, de convivencia y forma de vida. La palabra crisis en griego significa cambio, no por ello debe el cambio significar un empeoramiento de la situación, pero siempre las crisis van acompañadas de un proceso de adaptación y esos cambios implican esfuerzos, cuando no renuncias, de las partes implicadas. La educación está sufriendo este proceso y cuanto antes nos adelantemos al cambio antes saldremos de esta crisis. Mientras que los avances científicos, tecnológicos y sociales marcan la realidad actual, los modelos educativos siguen anclados en patrones instructivos y trasmisores, más propios de la era industrial. Como dice Richard Gerver profesor, escritor y asesor educativo británico "La labor del profesor es cambiar la forma en la que los alumnos ven el mundo y ayudarles a desplegar en él todo su potencial. Por eso, para que la educación cambie los primeros que deben salir de su zona de confort y los primeros que deben cambiar son los profesores" (Gerver 2012: 5).

La tarea de educar se ha hecho cada día más exigente y compleja. Las transformaciones sociales, la expansión del conocimiento y de los medios de comunicación, así como la competencia económica dentro del mundo globalizado, ejercen gran presión sobre la escuela, ya que preparar a los alumnos del presente y del futuro se ha convertido en una prioridad. Los países que han logrado el progreso de toda su población han invertido en educación. La mejor manera de superar la crisis con la mirada puesta en el futuro es proporcionar una formación integral, desarrollando los potenciales propios de cada alumno, haciendo hincapié en la creatividad y la actitud emprendedora.

Esta tarea no resulta sencilla de realizar con los sistemas dominantes y con los planteamientos sindicales y funcionariales que anteponen sus intereses a un cambio en la concepción docente. Uno de los pilares fundamentales para adecuar la enseñanza a los nuevos tiempos radica en la formación inicial y continuada del profesorado. Junto a los contenidos objeto de instrucción, se ha de formar en competencias didácticas, en

\footnotetext{
${ }^{1}$ Profesora de ciclos formativos de turismo del año 2004 en l'Escola d'Hoteleria i Turisme de Barcelona.
} 
actitudes creativas y el desarrollo de una nueva conciencia. Que el profesorado sea consciente de la importancia de su labor y la realice con ilusión, respeto y entrega para sacar lo mejor de cada uno de sus alumnos.

Son diversos los motivos por los cuales la profesión docente entraña mayor complejidad que cualquier otra, sobre todo en estos momentos en los que los cambios afectan a tantos aspectos de la sociedad. Algunos de los motivos son:

a) Los acelerados cambios en la sociedad, en los avances científicos y la construcción del conocimiento, en las demandas sociales a la escuela, en la incorporación masiva de las tecnologías de la información y la comunicación.

b) El sistema educativo va evolucionando a un ritmo muy lento, abriéndose una brecha cada vez mayor entre los programas y currículos instructivos y los intereses de los estudiantes y sus formas de vida.

c) La fragmentación del conocimiento que disciplinas separadas que reclaman docentes especializados, olvidando que la clave no está en los contenidos instructivos, sino en las personas. Esa fragmentación sólo existe a nivel académico, pues en la práctica de la vida los saberes están interconectados.

d) Los continuos recortes en educación, aumento de horas y empeoramiento de condiciones laborales hace que este oficio sea cada vez más precario. No deja de ser un mito en la mayor parte de los casos la referencia al profesorado como aquel que tiene un trabajo fácil, bien remunerado, horario reducido y largas vacaciones.

La enseñanza pública, que está considerada como una función esencial del Estado, es un poderoso instrumento que ayuda a la igualdad social, a través de ella podemos tener una sociedad más justa y con las mismas oportunidades para todos; pero para ofrecer una enseñanza de calidad y adaptada a los tiempos que corren se debe que tener en cuenta que la clave está en tener un profesorado bien formado, implicado en la causa, con vocación, con aptitudes, actitudes y sobre todo y ante todo motivado y con ganas de llevar a cabo su labor. Desde 1980 se han sucedido cinclo cambios legislativos en materia de educación y parece ser, que a la luz del Informe del Programa Internacional para la Evaluación de los Estudiantes, basado en el rendimiento de los estudiantes a partir de unos exámenes realizados cada tres años con el fin de determinar la valoración internacional de los alumnos, el rendimiento general ha mejorado muy levemente que pese a los esfuerzos de las instituciones para mejorarar los resultados. Hay que diseñar la educación pensando en los alumnos, que son los verdaderos protagonistas, en vez de pensar en lo que nos conviene como adultos o lo que nos gusta como profesores. Pero, además, los estudiantes deberían tener la palabra cuando se habla de educación, cuando se debate sobre su futuro, para dar a conocer sus aspiraciones, sus sueños y sus valores y lo que esperan del sistema educativo (R. Geber ,2012:67)

La mejora y la calidad de la enseñanza no viene dada exclusivamente por los recursos materiales disponibles en el centro, ni por lo elevado del salario, ni por la ratio alumnos/profesor, sino en parte por la toma de conciencia de la importancia de su papel en la educación, en tanto que estimulador de los potenciales de los alumnos. No se trata tanto de trasmitir cuanto de sacar, de despertar y seducir. Eso es precisamente la riqueza de la educación. Esta toma de conciencia conlleva una reflexión interior para conocerse mejor y conocer a sus alumnos. Conocer sus teorías implícitas, sus impulsos básicos, preferencias personales o patrones de comportamiento construidos a base de saberes y experiencias, conocer su estilo de evaluación que explica las diferencias entre los docentes. Todo ello es lo que determinan muchas de sus prácticas y modos de comportarse en el aula y fuera de ella. Y de esto va, precisamente, nuestra investigación.

\section{SENTIDO Y FUNDAMENTACIÓN}

Los profesores que imparten ciclos formativos de Turismo se dedican a formar profesionales del mañana, en un país en el que este sector es uno de los principales motores económicos. El peso económico que tiene el turismo en nuestro país es un 11,1\% del Producto Interior Bruto, según los datos del 2016 ofrecidos por el Instituto Nacional de Estadística en la Cuenta Satélite del Turismo de España y un 13\% del total de los puestos de trabajo nacionales según datos del 2016 del Instituto Nacional de Estadística. Pese al peso económico y el elevado porcentaje de ocupación laboral se puede afirmar que no existe a día de hoy ningún estudio publicado en España que planteé el pensamiento del profesor de ciclos formativos de turismo. El motivo de esta investigación es reflejar el pensamiento de este colectivo en relación con su labor docente. Cabe mencionar que la mayor parte de trabajos publicados en esta línea se centran en la enseñanza obligatoria ya que en este periodo de la escolarización hay una extensa bibliografía, especialmente sobre los procesos de enseñanza-aprendizaje en los módulos profesionales. 
Tres conceptos se consideran relevantes para fundamentar el trabajo aquí expuesto: la propuesta pedagógica de sentir y pensar del profesorado, las matrices pedagógicas, las teorías implícitas del profesorado.

"El pensamiento del Profesor" se convirtió en la década de los 80 del pasado siglo en un verdadero paradigma en la construcción del conocimiento por cuanto significó una ruptura del paradigma proceso-producto que es el que predominaba en las ciencias Sociales y en Pedagogía desde inicios del s. XX. Los trabajos de J. Gimeno, A. Pérez, S. Guerra, J.M. Escudero, entre otros, contribuyeron a poner el foco de atención de la investigación educativa en lo que ocurre en la mente de los profesores más que en las conductas. Los cambios externos no son sino una manifestación de lo que ocurre en el interior de las personas. Por ello, apareció una corriente que focalizó su atención en las creencias, actitudes, valores y pensamientos de profesorado y alumnado como elementos determinantes de las conductas externas. Las Universidad de Sevilla, Málaga, con investigadores como C. Marcelo, L. M. Villar (1998), Serrano (2007) y otros han llamado la atención sobre el pensamiento del profesorado en general y del profesorado novel en particular. Cómo van construyendo sus esquemas pedagógicos y didácticos a través de las teorías y las experiencias. Cómo las creencias y convicciones influyen y determinan el tipo de prácticas.

No se trata tan solo de averiguar qué piensa u opina el profesorado, sino qué siente, cuál es su sistema de creencias, sus preocupaciones, aspiraciones e insatisfacciones. Saber algo más sobre sus teorías implícitas y sus impulsos básicos a partir del cuestionario de preferencias personales o estilos de vida (Torre, 2006). Tanto el pensar como el actuar ocurren en el espacio determinado por las emociones. Cuáles son sus impulsos básicos que subyacen en buena parte de los comportamientos y que marcan un estilo de vida, un estilo de enseñanza y un estilo u otro de evaluación

Matrices pedagógicas. En diversas investigaciones realizadas por (Furlanetto, 2011:129) se constata que el profesorado al entrar en contacto con las prácticas pedagógicas y sus trayectorias formativas, se conectan con sus dimensiones vivenciales, pero también con las invisibles y desconocidas de la formación. A decir de dicha autora que viene investigando sobre matrices pedagógicas y formación docente, cada profesor posee una especie de profesor interno (patrones mentales de sus vivencias escolares) que emerge cuando ejercita su docencia. Para entender este proceso o mecanismo se ha construido el concepto de "matriz pedagógica" con el intento de comprender mejor las dimensiones de la docencia que permanecen a la sombra, pero que influyen o interfieren en su modo de actuar. Las "matrices pedagógicas" pueden ser comparadas a los archivos existenciales que guardan registros sensoriales, emocionales, cognitivos accediendo a ellos a través de situaciones o escenarios de la práctica pedagógica. (Furlanetto, 2011:129).

Teorías implícitas. El concepto de "teorías implícitas" (también llamadas espontáneas, causales o intuitivas) se viene utilizando en el ámbito de la ciencia y la Psicología para referirse a una estructura compleja organizada a ideas prototípicas en un mismo nivel. Para autores como Rodríguez y González (1995) y Pozo (1996), procedentes del ámbito de la Psicología son conexiones entre unidades de información aprendidas implícitamente por asociación, a partir de experiencias en el seno de grupos sociales reducidos próximos al individuo. Las teorías implícitas se caracterizan por basarse en información de tipo episódico o autobiográfico, ser muy flexibles frente a las demandas o situaciones en que son utilizadas y presentar ciertas normas 0 convencionalismos en sus contenidos, los cuales representarían los del grupo social al cual pertenecería el individuo (Linostroza y Quijada, 2008).

Para el pedagogo y didacta Marrero Acosta (2007) las teorías implícitas se abstraen, principalmente, a partir de un conjunto de experiencias almacenadas en la memoria. Se trata de un modelo de aprendizaje de teorías basado en la adquisición de "ejemplares" o "experiencias de conocimiento directo del objeto" y en la información comunicada lingüísticamente "experiencia de conocimiento directo". Las teorías educativas, no son productos cognitivos conscientes, resultado de una elaboración explicita por parte de los profesores. Se trata de síntesis que permanecen habitualmente implícitas. Este hecho queda bien reflejado en aquella frase que se escuchaba en otro tiempo "Cada maestrillo tiene su librillo" que no es otra cosa que una manera de entender y concebir la enseñanza y forma de llevarla a la práctica. De ahí que, aunque se pueda hablar de "teorías del profesorado" en general, de hecho, cada uno tiene sus propias teorías implícitas o formas gestionar los programas, tiempos, estrategias y actividades del alumno y la evaluación de los aprendizajes.

Si esto es así, ¿qué ocurre con el profesorado novel que no ha llegado a construir ese sistema de creencias? En buena lógica carecería de dicho sistema, sobre todo por lo que se refiere a su práctica. Se ha comprobado, escribe E. Rodríguez citando a Marcelo, que los profesores principiantes se encuentran con ciertos problemas específicos de su estatus profesional: imitación acrítica de conductas observadas en otros profesores, aislamiento de sus compañeros, dificultad para transmitir el conocimiento adquirido en su etapa de formación y desarrollan una concepción técnica de la enseñanza. De ese modo vemos la relevancia de que el profesorado vaya construyendo sus propias teorías y se le ayude a ello a través de la formación continua.

Resumimos en el siguiente diagrama las principales funciones de un profesor hoy. Como vemos son múltiples y diversificadas, de tal modo que el perfil que se deriva de esta propuesta, contrasta con su escaso reconocimiento social y prestigio. 


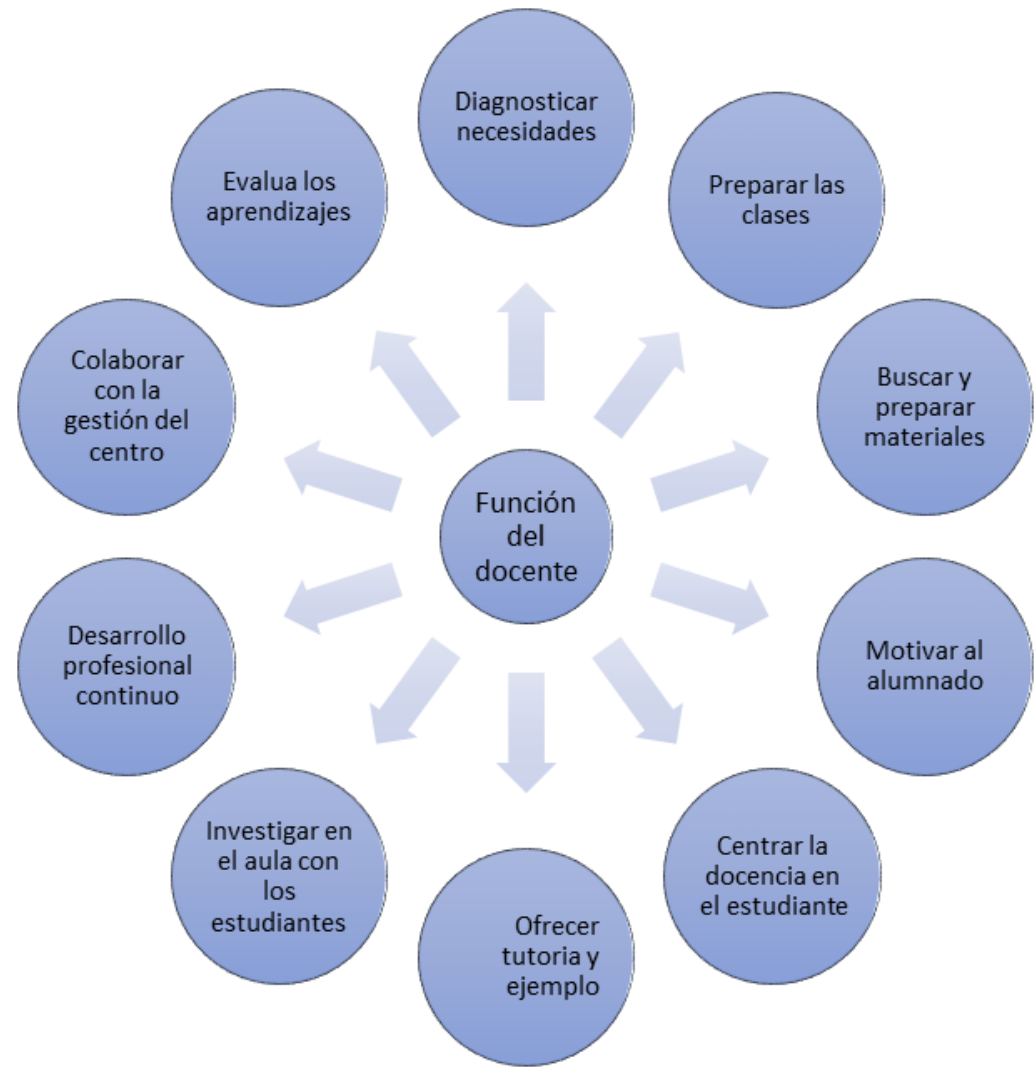

Gráfico 1: Funciones del docente

Fuente: Creación propia

\subsection{Problemática y objetivos}

La educación no escapa a la problemática general y a los cambios económicos, políticos, sociales y laborales que afectan a la sociedad actual, incluidos los cambios en valores. El profesorado se resiente en cierto modo de un continuo vaivén de propuestas políticas que no terminan por reconocer la importancia de su labor en la mejora de la sociedad. La sociedad futura está en sus manos y, sin embargo, no se lo tienen en cuenta en las reformas educativas, en los cambios curriculares, en las normativas administrativas. La Administración acrecienta sus exigencias y congela sus salarios, no siendo reconocido en sus esfuerzos formativos, en la dedicación innovadora, en la consideración social. La sociedad valora más a cualquier profesional que aun profesor de enseñanza obligatoria. $Y$ esto contribuye a un desencanto, sobre todo cuando es presionado por la Administración, los padres y los alumnos.

Si nos centramos en la enseñanza profesional, el abandono y marginación administrativa es aún mayor. Por desgracia no se la ha considerado como una formación relevante en el tejido sociolaboral, como ocurre en otros países; por el contrario, se la ve como una salida honrosa para estudiantes desmotivados hacia una enseñanza universitaria y sin perspectiva ocupacional inmediata. Sin embargo, nutre el ámbito productivo de excelentes profesionales, con menor coste para la sociedad. Basta considerar el nivel de paro universitario y contrastado el de los titulados de módulos profesionales y más concretamente en el sector del turismo y ver según datos del 2015 ofrecidos por la Cámara de Comercio de Cataluña el 82,17\% de los alumnos graduados han encontrado trabajo en relacionado con la formación recibida versus un $63,3 \%$ de los alumnos que han cursado el grado universitario de turismo, según datos ofrecidos por la Universidad de Barcelona.

Conocer la realidad actual de los centros, programas, profesorado, innovación y mejora, así como centrados en alumnado. Las principales intenciones de la investigación son la de constatar el pensar y sentir del profesorado de ciclos formativos de turismo, así como conocer sus inquietudes, preocupaciones e importancia que otorgan a la formación recibida.

Los objetivos específicos que planteados en el trabajo son:

1. Constatar el grado de satisfacción del docente con respecto al ambiente laboral y el ejercicio de su profesión.

2. Conocer cómo son percibidas las condiciones laborales de los docentes de CF.

3. Saber su opinión sobre el acceso a la función docente.

4. Constatar su opinión sobre la formación inicial y continuada. 
5. Conocer cómo perciben la relación entre el profesorado y el mundo de la empresa.

6. Percepción de la desmotivación del alumnado.

7. Recoger la opinión del profesorado respecto a las metodologías utilizadas en el aula.

8. Constatar los cambios producidos en su profesión.

\subsection{Metodología de investigación}

Se plantea un estudio exploratorio intentando buscar elementos y claves que nos permitan conocer mejor el pensar, sentir del profesorado de los ciclos formativos de turismo en centros de titularidad pública y por ello recurrimos a una metodología descriptiva. Ella se ajusta mejor a los objetivos pretendidos y es coherente con el paradigma interpretativo del pensamiento del profesor.

Este intento de captar las dimensiones del pensamiento y la posible evolución de las creencias de los profesores se llevó a cabo mediante un cuestionario online a profesores que trabajan en Centros públicos de ciclos formativos de turismo en Cataluña. Las variables que se someten a análisis son: el sexo, la edad, profesores noveles y experimentados y categoría laboral. La muestra quedó reducida a 25 profesores encuestados en noviembre de 2016, esta cantidad equivale a una muestra del $19,3 \%$ del total de profesores de ciclos formativos de turismo. Según datos aportados por del Departament d'Ensenyament de la Generalitat de Catalunya durante el curso 2016-17 hay en activo un total de 129 profesores de CF de Turismo en centros públicos en Catalunya. El siguiente cuadro resume las variables indicadas:

Edad: Entre los 40 y 50 años, el 36\%, entre 30 y 40 con un $28 \%$, entre 20 y 30, entre 50 y 60 años ,60 a 67 años con un $8,3 \%$.

Sexo: $91,7 \%$ mujeres $12 \%$ hombres

Antigüedad: entre 6 y 10 años 33,3, entre 11 y 15 un 33,3\%, entre 2 y 5 años, un 24,2\% y por último y los de menos de dos años un $4,2 \%$

Categoría laboral: $30,4 \%$ funcionarios sin plaza, interinos un $26,1 \%$, los funcionarios con plaza un $26,1 \%$, los sustitutos que representan un $17,39 \%$.

Tabla 1: Resultado encuesta

Fuente: Creación propia

\subsection{Análisis de resultados}

Siguiendo los ítems del cuestionario online obtuvimos los siguientes resultados.

1. Motivación a la carrera docente. El nivel de éxito y satisfacción profesional está relacionado con los motivos que le llevaron a dicha actividad. Es lo que Ken Robinson llama "el Elemento"; esto es, el hacer coincidir las competencias con la inclinación emociona y el aprovechamiento de las oportunidades. Así, cuando la elección de la carrera docente viene motivada por un verdadero interés educativo, tenemos muchas probabilidades de que confluyan el pensar, sentir y actuar de forma coherente y constructiva.

\begin{tabular}{lc}
\hline MOTIVACIÓN HACIA LA CARRERA & PORCENTAJE \\
\hline Interés por la enseñanza & $37,50 \%$ \\
\hline Condiciones laborales & $25,00 \%$ \\
\hline Otros motivos & $37,50 \%$ \\
\hline
\end{tabular}

Tabla 2: Motivación carrera docente Fuente: Creación propia

a) El porcentaje de profesores que se sienten atraídos por la enseñanza es equivalente al que llegan a ella por otros motivos no vocacionales, como las condiciones laborales, de funcionariado, las largas vacaciones o poder trabajar cerca de casa. El profesorado de módulos profesionales de turismo no es de los más motivados, lo cual se traduce en que el foco de su actuación no es el alumno, sino desarrollar el programa.

b) Pertinencia del proceso de selección. La opinión de los docentes sobre el proceso de selección del profesorado no es el adecuado. La mayoría de los encuestados considera que no es adecuado (33,3\%) o que lo es sólo en parte $(23,8 \%)$, frente al 42,9 que lo considera adecuado. El sistema está fallando a juicio del profesorado a la hora de seleccionar a los mejores profesores.

c) Públicos o privados. La mayor parte del profesorado ha trabajado en centro públicos (60\%) frente a un $40 \%$ que han trabajado en el privado. Aunque existe una corriente crítica respecto a la enseñanza pública, principalmente por parte de profesionales de la educación, el profesorado sigue prefiriendo trabajar la enseñanza pública. La critican, sí; pero a la hora de elegir, la prefiere a la privada por su libertad, autonomía, y condiciones. 
d) Formación inicial. El pensamiento del profesorado de ciclos formativos de turismo no está satisfecho de la formación recibida. No deja de sorprender que solamente un 4,4\% está totalmente satisfecho, mientras que un $52 \%$ la considera insuficiente y un $43,6 \%$ suficiente sólo en parte. Ello nos lleva a pensar que las instituciones universitarias que realizan tal formación, no cubren las expectativas del profesorado de ciclos formativos.

e) Formación continua. Un 75\% afirma llevar a cabo cursos de formación, mientras que un $25 \%$ confiesa que lo hace formación. Ello es coherente con la necesidad de completar la formación no recibida y que, dados los tiempos cambiantes, es preciso reciclarse. Por tanto, podríamos hablar de que existe una conciencia profesional de adecuarse a los nuevos tiempos.

f) Relación entre formación y trabajo. Las respuestas están muy dispersas y contrapuestas en esta cuestión. Un $36,4 \%$ ha indicado que sí, un $36,4 \%$ que en parte y un $27,36 \%$ que no. Tratándose de módulos profesionales resulta llamativo que no encuentren una relación entre la formación recibida y el trabajo. Que ocurra en otras carreras más teóricas y humanísticas, puede entenderse, máximo en tiempo de crisis. Entendemos que lo que motiva estos resultados son esas creencias extendidas en la profesión docente entre posicionamientos más ideales y aquellos que opinan desde la realidad conocida. Son respuestas que obedecen a la matriz pedagógica de la propia experiencia más que a datos reales.

g) Relación entre mundo laboral y docencia en CF. La presente cuestión esclarece y cambia la perspectiva cuando se trata de establecer una relación entre la aproximación de los alumnos al mundo laboral y su rol docente. El profesor es parte implicada en este proceso formativo y el $90 \%$ entiende que existe esa relación, quedando un $10 \%$ residual que adoptan una postura contraria. Conocedora de esta realidad por desempeñar precisamente la función de conexión con la empresa, tengo que afirmar que todo alumno de módulos profesionales tiene que llevar a cabo una estancia en la empresa como formación práctica.

h) Profesor especialista vs. Generalista. Desde el bachillerato a la universidad prevalece la cultura de la especialización por parte de los docentes. Un profesor generalista parece corresponderse mejor con una enseñanza básica y obligatoria. Luego prevalecen los conocimientos sobre la formación de la persona y consideran que el profesorado debe ser especialista en una determinada materia o disciplina. La realidad es bien otra. Un profesor interino puede exigírsele que imparta docencia de cualquier asignatura del currículo en cualquier curso. La especialización parece que afecta especialmente al profesorado estable. Por otra parte, las directrices europeas remarcan la necesidad de una formación diversificada y flexible para que la adaptación al mundo laboral sea mejor. Desde esa política se acortaron las carreras a cuatro años haciéndolas más generalistas y dejando los másteres para la especialización. En nuestro estudio, el $85 \%$ de los preguntados considera que es mejor que los docentes se especialicen en pocas materias y dominarlas a fondo frente a un $15 \%$ que considera que es mejor ir variando. Es evidente que una parte de ese $85 \%$ se mueve por intereses personales más que del alumno.

i) Metodología didáctica. Entrando en su práctica en el aula, se les pregunta por la adecuación de la metodología didáctica utilizada. En esto hemos de reconocer su sinceridad, salvo que estén pensando en el resto del profesorado y no en sí mismos. Lo cierto es que sólo el $22 \%$ afirma que se utiliza una metodología adecuada, frente al $30 \%$ que dice que no y un $48 \%$ que afirma solo en parte. La interpretación que hacemos de estos resultados es que está predominando una metodología transmisiva e informativa y muchos de ellos tienen una conciencia de que debería ser más activa, implicativa y constructiva haciendo uso de las nuevas tecnologías yd e espacios flexibles o fuera del aula, aspectos estos que no se favorecen. Antes, al contrario, se ha llegado a desalentar a algunos profesores por sacar a los alumnos fuera del centro.

j) Desmotivación del alumnado. En esta cuestión se dispersan nuevamente los pareceres. Mientras que un $48 \%$ no reconoce tal desmotivación, tan sólo un $12 \%$ afirma que sí existe y un $40 \%$ que en parte. Entendemos que inspiran tales respuestas la experiencia personal con los alumnos más que una opinión fundamentada. Es evidente que existe una ola de insatisfacción y desmotivación del alumnado, en general, tanto por los contenidos de los programas como por la metodología y evaluación. Pero en nuestro caso no se confirma esa tendencia, al menos estadísticamente. Para motivar a los estudiantes y para educarles de verdad debemos ofrecerles un aprendizaje que les importe, que les afecte, les divierta y les apasione, que signifique algo para ellos aquí y ahora, no para el futuro ni para preparar un examen. La tarea de los profesores ya no consiste en transmitir información y conocimientos a los alumnos, sino en ser capaces de unir lo que interesa a sus alumnos con las habilidades que necesitan desarrollar, y crear así un entorno de aprendizaje significativo y motivador para ellos.

k) Formación personal e interactiva. Los estudios científicos confirman la importancia de la relación humana, tanto en la educación, como en la salud y servicios sociales. El ser humano es sensible al trato y a la confianza que se le 
ofrece. Un $60 \%$ cree que siempre mejora la formación de cara al estudiante una formación más personal e interactiva. Un $35 \%$ considera que en ocasiones si y casi un $5 \%$ cree que nunca. Es evidente la influencia cultural sin duda combinada con la propia experiencia. Si bien es importante la formación, lo realmente efectivo es llevarlo a la práctica con los alumnos. Hay muchas sociedades, comunidades, clases y alumnos diferentes, por lo que la educación no puede ser igual para todos, no puede ser un sistema único e inamovible, sino que debe cambiar y personalizarse. El nuevo sistema de educación que diseñemos debe ser flexible y adaptable a cada realidad y a las necesidades individuales de cada alumno.

I) Satisfacción en el ámbito laboral. Preguntados por el grado de satisfacción laboral, que viene a ser como la profesión en la práctica cotidiana, obtuvimos las siguientes respuestas que se distribuyen según la curva normal de Gauss. En los extremos de mucho y nada con un 11,1\%, lo cual evidencia la heterogeneidad de los sentimientos entre el profesorado de CF por lo que respecta a su trabajo. Un $56,6 \%$ dice que bastante y un $22,2 \%$ poco. Nos quedamos con el valor predominante que es superior al $50 \%$ que se muestran bastante satisfechos. Concluimos que en general, el profesorado se muestra satisfecho laboralmente, si bien no era la enseñanza la opción ideal, tal como vimos al inicio.

m) Intención de continuidad. Coherentes con ese grado de satisfacción, una mayoría no se plantea cambiar de trabajo. Un $71 \%$ tiene intención de continuar, reduciéndose a un $4,8 \%$ quienes no piensan seguir. Un elevado $23 \%$ contesta que no lo sabe. No deja de ser preocupante ese $23 \%$ muy elevado si lo comparamos con otras actividades profesionales. ¿Será que la enseñanza desgasta? O tal vez es el reflejo de la insatisfacción y desencanto de ese $11-22 \%$ ? Creemos que es esto último.

\section{CONSIDERACIONES FINALES Y CONCLUSIONES}

Sintetizando las aportaciones de esta investigación, destacaría las siguientes consideraciones y conclusiones, esperando ser contrastadas a través de entrevistas con mayor profundidad.

a) Es preciso incidir en la cualificación del profesorado de la formación turística, mejorando los procesos de selección e introducir mecanismos de formación permanente y de evaluación continuada del profesorado, con la finalidad última de captar a los/las profesionales más competentes es la manera de conseguir un sistema educativo de calidad. Sobre todo, identificar a aquellos que sienten pasión por la enseñanza, pues es esta pasión y entrega la que marca la diferencia entre unos y otros.

b) Resultaría deseable implantar una formación inicial acompañada antes de enfrentarse con el aula, ya que, aunque ahora el Certificado de Aptitud Pedagógica sea un requisito imprescindible para poder acceder a la bolsa de trabajo sería bueno que, en todos centros, no solo en los de referencia, se ofreciese al nuevo formador una asistencia inicial o la figura de un tutor que lo acompañase que le pudiera guiar al principio. Pienso que una de las claves para ofrecer una enseñanza de calidad es el hecho que el profesorado este no solo formado sino al día de las últimas tendencias en el mercado laboral ya que no olvidemos el objetivo de los ciclos formativos es ofrecer al estudiante unos para poderse introducir en el mercado laboral con el menor tiempo posible por ello creo que la formación debería ser obligatoria.

c) Las condiciones de trabajo de los profesores se han endurecido excesivamente y este hecho tiene como consecuencia una merma en la calidad de las clases que imparte el profesorado ya que no dispone del tiempo ni los recursos deseados para poder ejercer bien su trabajo. Por otro lado, la adjudicación de tareas puramente administrativas que requieren mucho tiempo ayuda a restar tiempo del profesorado que podría dedicar a preparar las clases. Este hecho tiene un impacto directo con el grado de satisfacción del profesorado, que como ya se ha expuesto anteriormente en los resultados, el grado de satisfacción es bajo.

d) A la luz de los resultados expuestos en el trabajo se vislumbra un cierto acomodamiento propio del sistema funcionarial paralelamente a un de deseo de cambio, por parte especialmente de los docentes con menos años de ejercicio y con mayor precariedad laboral. Este deseo de cambio para la mejora de procesos o formas de operar viene acompañado en ocasiones de un sentimiento de impotencia dada la poca flexibilidad y rígida jerarquía del Sistema de enseñanza actual.

e) El sistema de oposiciones en la selección del profesorado de centros públicos adolece de varios inconvenientes y desde luego no garantiza elegir a los mejores profesores ni más capaces de desempeñar una 
función tan importante para la sociedad. Está orientado al dominio de conocimientos y estrategias, pero dice muy poco de la implicación, capacidad de trabajo en equipo y amor por la profesión.

f) El profesorado de ciclos formativos de turismo tiene una edad promedio de 41 años y una asentada experiencia docente. Podría ser una buena edad para la innovación a juicio del filósofo y sociólogo alemán Jürgen Habermas, porque ha llegado a adquirir las rutinas necesarias y pone su mirada en el desarrollo profesional.

g) El profesorado de nuestro estudio valora muy positivamente el contacto con el mundo empresarial. Tiene su razón de ser por cuanto los módulos de Formación están orientados al mundo laboral.

\section{ELENCO BIBLIOGRÁFICO}

Bisquerra. R. (coord) ,2012:" Metodología de la investigación educativa" La Muralla. 3를. ed., Madrid.

Clark, D. y Hollingsworth, H. ,2002: "Elaborating a model of teacher professional growth". Teaching and teacher education, Vol 18, 947-967.

Cruzado, Elisa ,2012: "Comunicación y atención al cliente en hostelería y turismo: certificado de profesionalidad: formación para el empleo" Mad, Madrid.

Damasio, A. ,2004: “ O erro de Descartes. Emoção, razão e cérebro humano”. Lisboa: Fórum da ciência.

Gerver, R. ,2012: "Crear hoy la escuela del mañana" SM, Barcelona.

Gómez-López, Javier y Cano, Jacobo ,2011: “El pensamiento docente y su influencia en las tecnologías de la información y la comunicación en el aula"Contextos educativos. 꾸 14, pp.

Lipton. B. ,2007: "Biología de la creencia" Almyra, Madrid.

Marcelo, C. (coord.),2011: "Evaluación del desarrollo profesional docente". Davinci. Barcelona.

Mcmillan, J. y Schumacher, S. ,2010:” Investigación educativa" Pearson. $5^{\text {a }}$ ed, Madrid.

Maturana, H. ,1999: "Transformación en la convivencia” Dolme,. Santiago.

Olmos. I. y García Cebrián, R. ,2012: "Cuerpo de profesores de enseñanza secundaria, hotelería y turismo. programación didáctica" Mad, Madrid.

Pozo J.I.,2008: "Aprendices y maestros" Alianza, Madrid.

Puelles M. de (coord) ,2009: "Profesión y vocación docente" Biblioteca Nueva, Madrid.

Robinson, K., 2011:" El elemento. descubrir tu pasión lo cambia todo" Debolsillo, Barcelona.

Serrano, R.C.,2007:" Pensamientos del profesor: un acercamiento a las creencias y concepciones sobre el proceso de enseñanza-aprendizaje". Revista Educación. oㅡ 352. pp.267-287.

Sola, M. ,2010:" El análisis de las creencias del profesorado como requisito de desarrollo profesional". Akal, Madrid pp. 661-683.

Torre, S.,2006:" Preferencias personales y estilos de vida, comprender y evaluar la creatividad" Aljibe. Vol 2. Málaga.

Tebar, I ,2003: "El perfil del profesor mediador" Aula XXI, Madrid.

Veal, A.J.,2006: "Research methods for leisure and tourism" Prentice Hall, London.

Villar, L.M. y otros ,2007: "Pensamientos de los profesores". Revista Bordón. Sociedad Española de Pedagogía № 49. pp. 5-14.

Zabalza, M.A.,2004: "Diarios de clase. un instrumento de investigación y desarrollo profesional” Nancea, Madrid. 